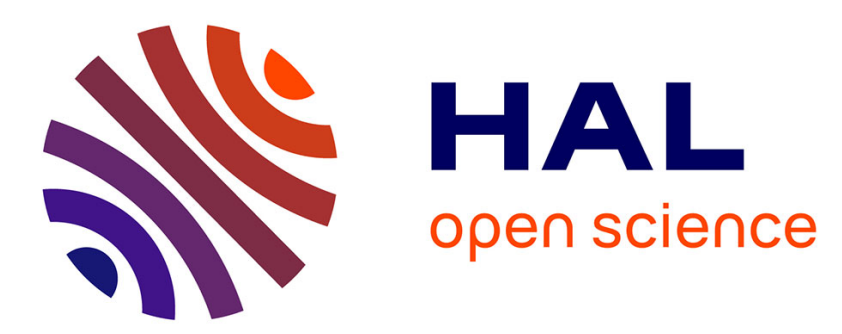

\title{
The boson character of spin-polarized atomic hydrogen demonstrated in magnetic relaxation
}

\author{
Ad Lagendijk, G.H. van Yperen, J.T.M. Walraven
}

\section{To cite this version:}

Ad Lagendijk, G.H. van Yperen, J.T.M. Walraven. The boson character of spin-polarized atomic hydrogen demonstrated in magnetic relaxation. Journal de Physique Lettres, 1984, 45 (19), pp.929933. 10.1051/jphyslet:019840045019092900 . jpa-00232433

\section{HAL Id: jpa-00232433 https://hal.science/jpa-00232433}

Submitted on 1 Jan 1984

HAL is a multi-disciplinary open access archive for the deposit and dissemination of scientific research documents, whether they are published or not. The documents may come from teaching and research institutions in France or abroad, or from public or private research centers.
L'archive ouverte pluridisciplinaire HAL, est destinée au dépôt et à la diffusion de documents scientifiques de niveau recherche, publiés ou non, émanant des établissements d'enseignement et de recherche français ou étrangers, des laboratoires publics ou privés. 
Classification

Physics Abstracts

$05.30 \mathrm{~J}-67.20 \mathrm{~K}$

\title{
The boson character of spin-polarized atomic hydrogen demonstrated in magnetic relaxation
}

\author{
Ad Lagendijk, G. H. van Yperen and J. T. M. Walraven \\ Natuurkundig Laboratorium der Universiteit van Amsterdam, Valckenierstraat 65, \\ 1018 XE Amsterdam, The Netherlands
}

(Reçu le 4 juillet 1984, accepté le ler août 1984)

\begin{abstract}
Résumé. - Nous démontrons que le taux de relaxation magnétique dans l'hydrogène atomique à spin polarisé contient une correction d'échange importante. Une comparaison avec les observations expérimentales met clairement en évidence le caractère bosonique de l'hydrogène atomique. Nous avons calculé les taux de recombinaisons pour les processus $b \rightarrow a$ et $b \rightarrow c$.
\end{abstract}

\begin{abstract}
We demonstrate that the spin relaxation rates in gaseous spin-polarized atomic hydrogen exhibit large exchange corrections. The experimentally observed nuclear relaxation rates should be considered as a clear experimental proof of the boson character of atomic hydrogen. We have calculated the relaxation rates for the $b \rightarrow a$ and for the $b \rightarrow c$ processes.
\end{abstract}

Spin-polarized atomic hydrogen $(\mathrm{H} \downarrow)$ is commonly envisioned as probably the purest example of a weakly interacting Bose gas [1]. Employing the well-known Ehrenfest-Oppenheimer argument one infers that $\mathrm{H} \downarrow$ should obey Bose statistics because it consists of an even number of tightly bound fermions [2]. As the most spectacular features of quantum many-body systems show up in the neighbourhood of their degeneracy temperatures much experimental effort is focused on increasing the density of this gas up to values where Bose-Einstein condensation should occur. Although the statistical nature of the gas is of obvious importance to the experimental activity it is remarkable to note that to our knowledge no experimental evidence for the boson nature of $\mathbf{H} \downarrow$ exists, yet the limits of validity of the statements concerning the statistical nature of composite particles has attracted theoretical attention over the years, both for the general case as well as in particular for the H-atom [3-6].

In this Letter we analyse the requirements for the boson nature of $\mathrm{H} \downarrow$ to show up in experiments. We compare the theory of magnetic dipolar relaxation with experiment and show that the current level of experimental accuracy is adequate to establish the bosonic nature of $\mathrm{H} \downarrow$. We find that the indistinguishability of the $\mathrm{H} \downarrow$ atoms gives rise to a large exchange correction which is in addition insensitive to the exact form of the potential over a wide range of temperatures. These features make dipolar relaxation a very clean property, in contrast to potential scattering, where the details of the potential are important.

$\mathrm{H} \downarrow$ is a gas with atoms in two hyperfine states : the mixed ground state $|\mathrm{a}\rangle=-|\downarrow \uparrow\rangle+$ $\varepsilon|\uparrow \dot{I}\rangle$ and the pure spin state $|\mathrm{b}\rangle=|\downarrow I\rangle$ (here $\uparrow$ and $\uparrow$ are electron and nuclear spin $1 / 2$ projections, and in high magnetic field, $\varepsilon \simeq a / 4 \mu_{\mathrm{B}} B$ is the hyperfine mixing parameter with $a$ the hyperfine constant and $B$ the magnetic field). The $b \rightarrow$ a relaxation rate in the gas phase was measured by several groups to good precision [7-10]. 
Recently the first experimental observation of $\mathrm{b} \rightarrow \mathrm{c}$ relaxation was reported ( $\mathrm{c}$ is an electronic « up » spin state $:|\uparrow I\rangle+\varepsilon|\downarrow \uparrow\rangle[10])$. The $\mathrm{b} \rightarrow \mathrm{c}$ relaxation is an important process, which has an exponential temperature dependence, and can put serious limitations on the conditions under which $\mathrm{H} \downarrow$ can be compressed to high densities.

Several authors have calculated the $\mathrm{b} \rightarrow$ a volume relaxation either in the Distorted-WaveBorn-Approximation (DWBA) [11, 12] or with a simple excluded-volume approach [13]. To analyse the dependence on statistics we calculated the $b \rightarrow a$ and $b \rightarrow c$ relaxation rates for both the boson and fermion case for $T<1 \mathrm{~K}$ using the excluded volume approach. In addition we have performed DWBA calculations to check the insensitivity of the results to the exact form of the potential. The only previous work with explicit numerical results and the inclusion of the finiteness of the energy separation between the a and b level is the work of Ahn et al. for bosons [12]. Our boson results are in good agreement with their work, but we have extended the calculations over a larger temperature range.

To observe phenomena associated with the statistical nature of (composite) particles one has to create the conditions in which quantum mechanical interference effects of properly symmetrized wavefunctions lead to different values for observables than for non symmetrized wavefunctions. The densities at which these exchange effects become important depend strongly on the specific observable being studied and more particularly with the length-scale which may be associated with the observable.

To observe deviations from the $p=n k T$ law in an ideal Bose gas one requires densities where the mean separation between the bosons is of the order of the thermal wavelength. For spin zero bosons with mass 1 this implies densities $n \gtrsim 6 \times 10^{18} / \mathrm{cm}^{3}$ for $T=100 \mathrm{mK}$. With real hydrogen atoms one has to be careful with such an analysis. In principle the statistical nature would also show up at lower densities as quantum corrections on the scattering contributions to the virial coefficients but these effects would be difficult to observe [14]. Clearly at densities where the electronic wavefunctions start to overlap $\left(n \gtrsim 10^{22} / \mathrm{cm}^{3}\right)$ electronic exchange will cause the picture to break down.

To search for the boson character of $\mathrm{H} \downarrow$ the thermodynamic state variables should not be the first choice. These properties do not depend on the interactions in zero'th order and rather high densities are required before exchange effects show up. Other properties only exist by virtue of the interactions. In these cases the properties depend critically on the nature of the scattering processes and indistinguishability effects may dominate the physics at much lower densities. As an example one may consider diffusion constants which show substantial exchange corrections but have the disadvantage that the results are sensitive to the exact form of the interatomic potential [15]. This potential has a very short range and causes plane wave approximations to be poor.

Magnetic dipolar $\mathrm{b} \rightarrow$ a relaxation is an ideal probe to search for the boson nature of $\mathrm{H} \downarrow$. The range of the dipolar interaction is in principle infinite though in practice finite due to the thermal motion of the atoms (motional narrowing). As a result for a large part of the temperature range of interest free particle motion gives already very accurate results. In the present context motional narrowing theory is equivalent to a scattering treatment. The physics of the motional narrowing process is that the translational motion is modulating in a stochastic way the dipolar interaction between particles. This process is described by the following correlation function,

$$
F_{i j}(t) \equiv\left\langle\frac{Y_{2}^{1}\left[\mathbf{r}_{i j}(0)\right]}{r_{i j}^{3}(0)} \frac{Y_{2}^{1}\left[\mathbf{r}_{i j}(t)\right]^{*}}{r_{i j}^{3}(t)}\right\rangle
$$

In definition (1) $\mathbf{r}_{i j}$ denotes the interatomic vector of particle $i$ and $j$. The relaxation rate $G_{b \rightarrow a}$ is proportional to the Fourier transform of $F_{i j}(t)$ at angular frequency $\Omega_{\mathrm{b} \rightarrow \mathrm{a}}$, the NMR frequency. In the excluded-volume approach $F_{i j}(t)$ is calculated using the basis $\operatorname{set}\left\{\mathrm{e}^{i \mathbf{k} . \mathbf{R}_{i j}} \mathrm{e}^{i \mathbf{q} \cdot \mathbf{r}_{i j} j}\right\}$, where $\mathbf{R}_{i j}$ represents the position of the centre of mass with momentum $\mathbf{k}$ ( $\mathbf{q}$ is the relative momentum of the 
pair), and excluding the region $r_{i j}<d, d$ the hard-core diameter (3.67 $\AA$ ). We emphasize again that for a large part of the results this exclusion is unnecessary and free motion is sufficient. In the DWBA approach the basis set $\mathrm{e}^{i \mathbf{k} \cdot \mathbf{R}_{i j}} Y_{l}^{m}\left(r_{i j}\right) R_{q l}\left(r_{i j}\right)$ is used, where $R_{q l}$ is the solution of the radial Schrödinger equation. Both calculations are straight-forward but tedious.

The identity of particles is taken into account by symmetrizing the wavefunction with respect to exchange of complete hydrogen atoms in well-defined hyperfine states, treating them either as bosons or as fermions. This picture breaks down when the concept of atomic hyperfine state is no longer valid, i.e. at small separation, where the electronic wavefunctions overlap. The latter occurs for atoms interacting via the singlet potential but for $\mathrm{b} \rightarrow$ a relaxation, where the triplet interaction prevents the atoms from getting close these effects are negligible. A rather complete discussion of the required approximations has been given by Ahn et al. [12]. In the calculation the difference between bosons and fermions is reflected in the occurrence of only even or only odd $l$ values in the matrix elements of $\left\{\boldsymbol{R}_{q l}\right\}$.

In figure 1 we present the results for $G_{\mathrm{b} \rightarrow \mathrm{a}}$ using the excluded volume approach and the DWBA for a magnetic field of $8 \mathrm{~T}$ and temperatures below $1 \mathrm{~K}$. In the same figure we have plotted the experimental data from several groups [7-10]. Neither in the excluded-volume approach nor in the DWBA the results can be presented in closed form.

Results at different magnetic fields can be scaled with the factor $\left(1+\varepsilon \gamma_{\mathrm{e}} / \gamma_{\mathrm{p}}\right)^{2}$. Scaling with this factor introduces an inaccuracy of $1 \%$ at $500 \mathrm{mK}$ which is slowly increasing to $7 \%$ at $20 \mathrm{mK}$ due to the dependence of the NMR frequency $\Omega_{\mathrm{b} \rightarrow \mathrm{a}}$ on the magnetic field.

Inspection of figure 1 demonstrates beautifully that the experimental data give very strong support for the boson character of $\mathrm{H} \downarrow$. Given the insensitivity of the results to the actual form of the potential we consider the nuclear relaxation rate in the gas phase ideally suited to probe the

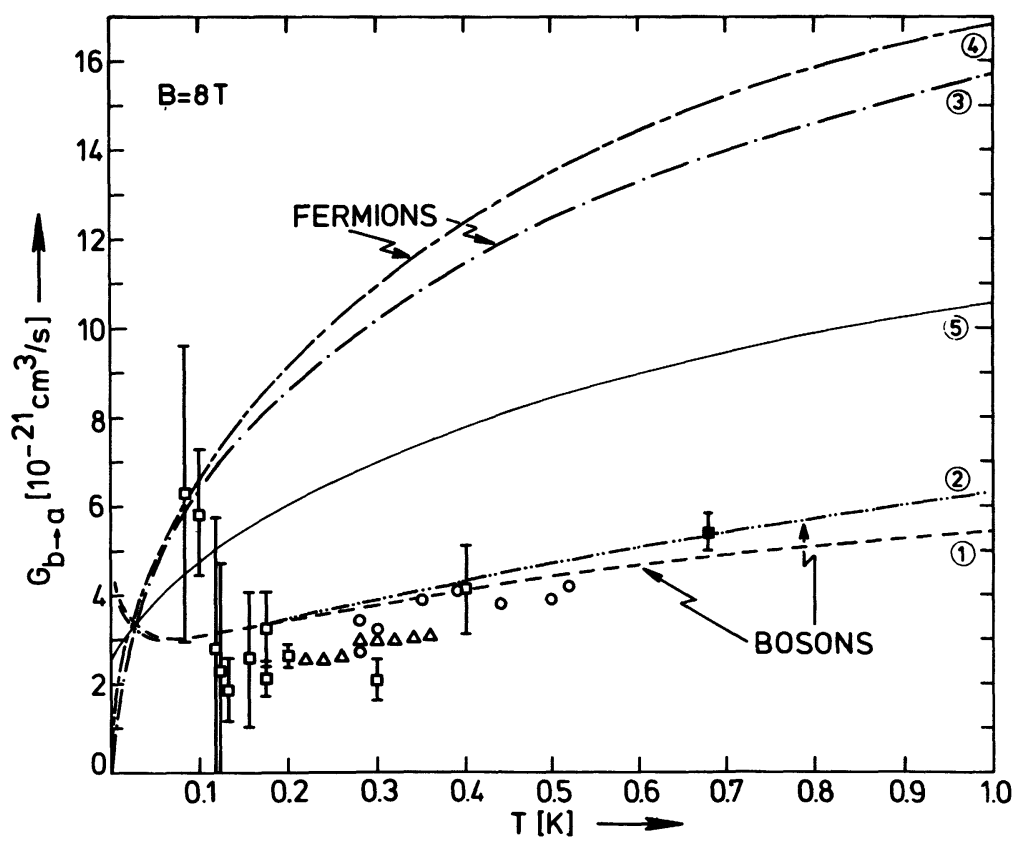

Fig. 1. - The volume nuclear relaxation rate $G_{b \rightarrow a}$ as a function of temperature for $B=8$ T. Calculations : (1) excluded-volume, bosons; (2) DWBA, bosons; (3) excluded-volume, fermions ; (4) DWBA, fermions ;

(5) excluded-volume, " classical ». Experimental results : $\Delta$ reference [7], $\square$ reference [8], $O$ reference [9], reference [10]. All results are scaled to 8 tesla where necessary. 


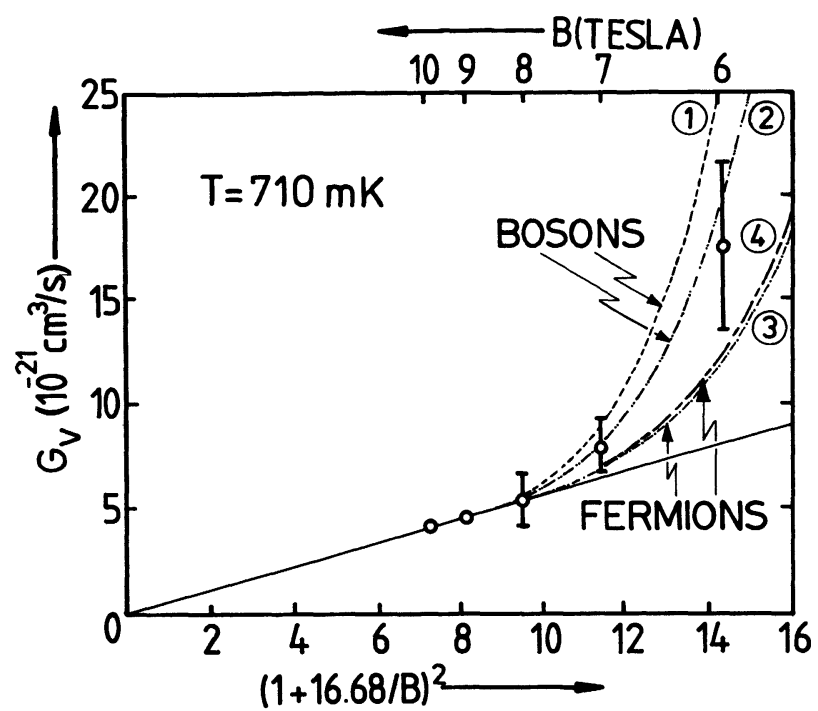

Fig. 2. - The volume relaxation rate as a function of magnetic field at $T=0.71 \mathrm{~K}$. Solid line : nuclear relaxation rate $G_{\mathrm{b} \rightarrow \mathrm{a}}$ (DWBA, bosons), lines 1-4 : electronic relaxation $G_{\mathrm{b} \rightarrow \mathrm{c}}$; the labelling is the same as in figure 1 . The experimental data are from reference [11].

boson character of $\mathbf{H} \downarrow$. Note that the data points tend to lie below the « boson » line. We believe this to be due to experimental inaccuracies.

Recently the $b \rightarrow c$ relaxation was observed [10]. This process has been discussed by Kagan et al. [16] but their results are not explicit enough to enable detailed comparison with experiments. We have calculated this process both in the excluded-volume approach and using the triplet potential. The difference between the two is about $30 \%$. It is interesting to note that there is a large exchange contribution $(\simeq 50 \%$ ) of opposite sign compared to the $b \rightarrow$ a relaxation. The DWBA results could be fitted to the form $G_{\mathrm{b} \rightarrow \mathrm{c}} \exp \left(g_{\mathrm{e}} \mu_{\mathrm{B}} B / k_{\mathrm{B}} T\right)=9.7 \times 10^{-16} \mathrm{~cm}^{3} \mathrm{~s}^{-1}$ in the temperature range around $0.71 \mathrm{~K}$ and magnetic field range $6 \mathrm{~T}<B<8 \mathrm{~T}$. The exponential dependence comes from the Boltzmann factor expressing the probability that high-energy fluctuations are present in the translational degrees of freedom. In figure 2 we plot the total depopulation rate of the $b$ level, which is the sum of the $b \rightarrow a$ and $b \rightarrow c$ rates, on such a scale that in the absence of $b \rightarrow c$ relaxation a straight line would result. The deviation of this straight line represents the $b \rightarrow c$ relaxation process. The experimental data of reference [10] are also shown in figure 2. It is clear that the data show the effect of the $b \rightarrow c$ relaxation. The accuracy of the experimental data is not sufficient to distinguish between Bose and Fermi behaviour.

\section{Acknowledgments.}

This research is supported by the Dutch Foundation for Research of Matter (F.O.M.).

\section{References}

[1] For a recent review : Silvera, I. F., Physica B 109 \& 110 (1982) 1499.

[2] Ehrenfest, P. and Oppenheimer, J. R., Phys. Rev. 37 (1931) 4.

[3] Pinard, M. and Lalö̈, F., J. Physique 41 (1980) 769.

[4] Girardeau, M. D., J. Math. Phys. 16 (1975) 1901.

[5] Freed, J. H., J. Chem. Phys. 72 (1980) 1414.

[6] Lefèvre-Seguin, V., Nacher, P. J., Lhuiller, C. et Lalö̈, F., J. Physique 43 (1982) 1199. 
[7] Cline, R. W., Greytak, T. J. and Kleppner, D., Phys. Rev. Lett. 47 (1981) 1195.

[8] Sprik, R., Walraven, J. T. M., van Yperen, G. H. and Silvera, I. F., Phys. Rev. Lett. 49 (1982) 153.

[9] Yurke, B., Denker, J. S., Johnson, B. R., Bigelow, N., Lévy, L. P., Lee, D. M. and Freed, J. H., Phys. Rev. Lett. 50 (1983) 1137.

[10] Sprik, R., Walraven, J. T. M. and Silvera, I. F., Phys. Rev. Lett. 51 (1983) 479, 942 (E).

[11] Statt, B. W. and Berlinsky, A. J., Phys. Rev. Lett. 45 (1980) 2105.

[12] Ahn, R. M. C., V. D. Eynde, J. P. H. W. and VerhaAR, B. J., Phys. Rev. B 27 (1983) 5424.

[13] Siggia, E. D. and Ruckenstein, A. E., Phys. Rev. B 23 (1981) 3580.

[14] Mello, E. V. L., Rehr, J. J. and Vilches, O. E., Phys. Rev. B 28 (1983) 3759.

[15] Lhuiller, C. and Lalö̈, F., J. Physique 43 (1982) 197 and 225.

[16] Kagan, Yu., Vartanyantz, I. A. and Shlyapnikov, G. V., Sov. Phys. JETP 54 (1981) 590. 